\title{
Can mesenchymal stem cell therapy be the interim management of COVID-19?
}

\author{
Chitra Bamba ${ }^{1}$, Surinder P. Singh ${ }^{2}$, Sangeeta Choudhury ${ }^{1, *}$ \\ ${ }^{1}$ Department of Research, Sir Ganga Ram Hospital, New Delhi, India; \\ ${ }^{2}$ CSIR-National Physical Laboratory, Dr K. S. Krishnan Marg, New Delhi, India.
}

SUMMARY COVID-19 pandemic has accounted for $\sim 4.3$ million confirmed cases and $\sim 292,000$ deaths (till $12^{\text {th }}$ May, 2020) across the globe since its outbreak. Several anti-viral drugs such as RNA dependent RNA polymerase inhibitors (remdesivir, favipiravir, ribavirin), protease inhibitors (lopinavir, ritonavir) and drugs targeting endocytic pathway (hydroxychloroquine) are being evaluated for COVID-19 but standard therapeutics yet not available. Severe health deterioration in critically ill patients is characterized by pulmonary edema, severe respiratory distress, cytokine storm and septic shock. To combat cytokine storm, immune-therapy targeting IL-1, IL-2, IL-6 and TNF $\alpha$ are being evaluated and one of the promising immune-modulator is the mesenchymal stem cells (MSCs) that can surmount the severity of COVID-19 infections. Recent studies have shown that MSC-therapy significantly dampens the cytokine storm in critically ill COVID-19 patients. This communication endows with the insight of stem cell therapy and summarizes the recent studies on COVID-19 patients.

Keywords novel coronavirus disease, SARS-CoV-2, cytokine storm, MSC-therapy

The entire world is in state of emergency due to outbreak of novel corona virus disease 2019 (COVID-19) that first reported in Wuhan, China in December 2019. It became a global health emergency due to non-availability of vaccine or specific therapeutic drugs. Till May 12, 2020 there were 4,337,602 cases worldwide among which 1,408,636 cases were from USA, making it an epicenter for this pandemic (as per worldometers.info). This ripple of COVID-19 has severely impacted the global economy and healthcare system. Currently the mainstay of COVID-19 management is symptomatic treatment and mechanical ventilation for critically ill patients. Medications include anti-viral drugs like remdesivir, ribavirin, favipiravir, lopinavir/ritonavir, hydroxychloroquine, and arbidol which blocks the entry of virus to host cell, and other drugs such as IFN- $\alpha 2 a$, IFN- $\beta 1 b$, tocilizumab which inhibits the viral exocytosis leading to regulation of immune response (1). However, till date, there's no COVID-19 approved drug available for the treatment. This necessitates a prodigious effort to understand the pathophysiology of the virus, develop efficient therapeutic modality as well as sensitive and specific rapid diagnostics in order to combat COVID-19 pandemic.

Coronaviruses $(\mathrm{CoV})$ belongs to the Coronaviridae family of viruses, which is subdivided into 4 classes i.e. alpha, beta, gamma and delta. The beta-coronavirus group includes Middle East Respiratory Syndrome (MERS-CoV), Severe Acute Respiratory Syndrome (SARS-CoV) and the novel SARS-CoV-2 the major causative agent for COVID-19 (2). The whole genome sequencing of SARS-CoV-2 shows a similarity of $96.2 \%$ to a bat betacoronavirus (SARSSr-CoV; RaTG13) while comparatively less similar to SARS$\mathrm{CoV}(\sim 79 \%)$ and MERS ( 50\%) (3). Similar to SARS$\mathrm{CoV}$ and MERS, SARS-CoV-2 attacks the respiratory tract causing severe respiratory distress, pulmonary edema and viral pneumonia. However, in severe cases, SARS-CoV-2 affects heart, kidney, liver, GI-system, leading to multiple organ failure and eventually death in some cases (4). SARS-CoV-2 is an enveloped, positive sense RNA virus with genome encoding various glycoproteins, including the glycosylated spike (S) protein. This S-protein binds to angiotensin I converting enzyme 2 receptor (ACE-2) in host cell. Also, ACE-2 receptor is highly expressed on the surface of lung alveolar type II cells (AT-2) and capillary endothelium. In addition, AT-2 cells also express type II transmembrane serine protease (TMPRSS211) which facilitates priming of S-protein and in turn the invasion of virus inside the host cell (5). Unfortunately, ACE- 
2 receptors are also expressed on other tissues like kidney, liver, heart and digestive system organs; thus explaining the rapid progression towards systemic inflammatory conditions as observed in critically ill patients. The silver lining is that thymus, bone marrow, spleen, lymph node and macrophages do not express ACE-2 receptors (6); this implies that exploiting immuno-therapeutic approaches to target SARSCoV-2 virus infection pathway can be feasible and may provide better treatment outcomes.

The clinical course of SARS-CoV-2 begins with robust viral replication accompanied with mild symptoms such as fever, cough, headache and the second phase exhibit high grade fever, difficulty in breathing and pneumonia-like symptoms. The progression to third stage is mediated by inflammatory cytokines (IL-2, IL-6, IL-8, TNF- $\alpha$, G-CSF, GMCSF), chemokines (MCP-1, MIP1 $\alpha$, IP10) and massive infiltration of inflammatory cells. This 'cytokine storm' is the hallmark of third stage SARS-CoV-2 pathogenesis in critically ill patients inducing pulmonary edema, dysfunction of air-exchange, acute respiratory distress syndrome (ARDS), cardiac injury and secondary infections which ultimately leads to death (7). A number of studies with drugs targeting GM-CSF, IL-6, IL-1, IL-2 and TNF- $\alpha$ is already in pipeline which aims to dampen the inflammatory response in COVID-19 patients. Mesenchymal stem cells (MSCs) are known for their immuno-modulatory properties such as secretion of anti-inflammatory cytokines/chemokines, anti-apoptotic effect and their ability to repair damaged epithelial cells. Their inherent nature to migrate towards injured lungs and secretion of paracrine factors which protects and repair alveolar cells; make MSCs a potential therapeutic option for COVID-19 treatment.

In recent years, MSCs have been widely employed from basic research to clinical trials $(8,9,10)$, especially for immune-mediated inflammatory diseases such as systemic lypus erythematous (SLE) (11) and graft versus-host disease (GVHD) (12). The success of MSCs is attributed to their ability to modulate immune response directly via interaction with host immune cells or indirectly through paracrine secretion of various cytokines $(8,13)$. MSCs modulate immune response by regulating the function and proliferation of various immune cells, inhibits monocyte differentiation into dendritic cells (DCs) which results in upregulation of regulatory cytokines and downregulation of inflammatory cytokines (14). Previous studies suggested that systemic administration of MSC resulted in reduction of $\mathrm{H} 5 \mathrm{~N} 1$ influenza virus-induced mortality in older patients with severe pulmonary illness (15). Also, in patients with H7N9 induced ARDS, a significant improvement in survival rate was observed (16). So far, MSC transplantation in human subjects with diverse disease conditions has not showed any severe adverse events (17). Therefore, it is plausible that MSC-therapy can be used to treat COVID-19 patients.

Recently, Leng $\mathrm{Z}$ et al. conducted a pilot study on 7 confirmed COVID-19 pneumonia patients presented with mild, common, severe and critically ill disease condition (18). Clinical grade MSCs were injected intravenously $\left(1 \times 10^{6}\right.$ cells $/ \mathrm{kg}$ body weight $)$ and followup for 14 days. A significant reduction in clinical symptoms and pneumonia infiltration was observed in chest CT of critically ill COVID-19 patient within 2-4 days of MSC-therapy. An increase in peripheral lymphocyte levels, decrease in C-reactive protein (CRP), drastic disappearance of activated cytokine-secreting immune cells (CXCR3+CD4+T-cells, CXCR3+CD8+Tcells and CXCR3+NK-cells) and restoration of

Table 1. Ongoing MSC-based clinical trials for COVID-19 treatment

\begin{tabular}{|c|c|c|}
\hline Cell source & Clinical trial number & Reference \\
\hline Human allogeneic MSCs (Source Unknown) & $\begin{array}{l}\text { ChiCTR2000030224 } \\
\text { ChiCTR2000030835 }\end{array}$ & http://www.chictr.org.cn \\
\hline & $\begin{array}{l}\text { NCT04288102 } \\
\text { NCT04299152 }\end{array}$ & https://clinicaltrials.gov \\
\hline Human Umbilical Cord derived MSC & $\begin{array}{l}\text { ChiCTR2000030866 } \\
\text { ChiCTR2000030138 }\end{array}$ & http://www.chictr.org.cn \\
\hline & $\begin{array}{l}\text { NCT04273646 } \\
\text { NCT04269525 } \\
\text { NCT04252118 }\end{array}$ & https://clinicaltrials.gov \\
\hline Wharton's Jelly derived MSC & $\begin{array}{l}\text { ChiCTR2000030088 } \\
\text { NCT04313322 }\end{array}$ & $\begin{array}{l}\text { http://www.chictr.org.cn } \\
\text { https://clinicaltrials.gov }\end{array}$ \\
\hline Dental pulp MSC & $\begin{array}{l}\text { ChiCTR2000031319 } \\
\text { NCT04302519 }\end{array}$ & $\begin{array}{l}\text { http://www.chictr.org.cn } \\
\text { https://clinicaltrials.gov }\end{array}$ \\
\hline Ruxolitinib in combination with MSCs & ChiCTR2000029580 & http://www.chictrorg.cn \\
\hline
\end{tabular}


regulatory DC cell population $\left(\mathrm{CD} 14+\mathrm{CD} 11 \mathrm{c}+\mathrm{CD} 11 \mathrm{~b}^{\mathrm{mod}}\right.$ regulatory DC cell) to normal levels was observed after day 6 of MSC transplantation. Additionally, an increased level of anti-inflammatory cytokine IL-10 and significantly decreased levels of serum pro-inflammatory cytokine TNF- $\alpha$ indicated efficientregulation of cytokine storm in COVID-19 patients on MSC transplantation. Furthermore, the absence of ACE-2 receptor and TMPRSS2 on the transfused MSCs affirmed that they cannot get infected with SARS-Cov-2, suggesting the usefulness of MSC-therapy in COVID-19 infection.

A case study reported that a $65 \mathrm{y} / \mathrm{o}$ type-II diabetic, hypertensive COVID-19 positive female with severe pneumonia, acute gastrointestinal bleed and ARDS was treated with MSCs (19). Initially, the patient did not respond to any anti-viral drug and thereafter progressed towards multiple organ injury. At this stage, the patient was intravenously injected with 3 doses of human umbilical cord MSC (hUC-MSC, $50 \times 10^{6}$ cells/dose). After second cell infusion, ventilator was removed as the vital stats had improved with gradual decrease in serum albumin and CRP levels. CT images showed no pneumonia patches by the end of MSC-therapy. These results suggest that hUC-MSC can be beneficial to patient who are resistant to anti-viral drugs. Owing to the therapeutic potential of MSCs in viral infections and its immense capability to regulate immune cells to ameliorate the cytokine storm, a few studies have been initiated to evaluate their efficiency for COVID-19 treatment (Table 1). Seeing the promising prospective of MSC-therapy and to meet the large-scale demand of cGMP produced stem cells, US-based biotech companies (Mesoblast, Athersys) have taken up the challenge for mass production of clinical grade MSCs (20).

In summary, it appears that MSC-therapy through its immuno-modulatory properties suppresses the over activated immune system and promotes the tissue repair of alveolar cells in lung microenvironment of SARSCoV-2 infected patients. Although the data derived from recent studies are encouraging, but the major limitations are the small-sized patient enrollment and thus necessitating larger randomized control trials to establish the effectiveness and safety of MSC-therapy in SARS-Cov-2 infection. It has also been observed that most of the clinical trials for COVID-19 treatment have used allogeneic stem cell source. With the vast available knowledge with reference to the mechanism of action of MSCs and their effective potencies at a specific disease stage makes MSCs as an ideal therapeutic candidate.

\section{References}

1. Dong L, Hu S, Gao J. Discovering drugs to treat coronavirus disease 2019 (COVID-19). Drug Discov Ther. 2020; 14:58-60.

2. Zhu N, Zhang D, Wang W. et al. A novel coronavirus from patients with pneumonia in China, 2019. N Engl J
Med. 2020; 382:727-733.

3. Tang X, Wu C, Li X, Song Y, Yao X, Wu X, Duan Y, Zhang H, Wang Y, Qian Z, Cui J, Lu J. On the origin and continuing evolution of SARS-CoV-2. Natl Sci Rev. 2020.

4. Tang B, Bragazzi NL, Li Q, Tang S, Xiao Y, Wu J. An updated estimation of the risk of transmission of the novel coronavirus (2019-nCov). Infect Dis Model. 2020; 5:248-255.

5. Hoffmann M, Kleine-Weber H, Schroeder S, Krüger N, Herrler T, Erichsen S, Schiergens TS, Herrler G, Wu NH, Nitsche A, Muller MA, Drosten C, Pohlmann S. SARSCoV-2 cell entry depends on ACE2 and TMPRSS2 and is blocked by a clinically proven protease inhibitor. Cell. 2020; 181:271-280.

6. Hamming I, Timens W, Bulthuis MLC, Lely AT, Navis GJ, van Goor H. Tissue distribution of ACE2 protein, the functional receptor for SARS coronavirus. A first step in understanding SARS pathogenesis. J Pathol. 2004; 203:631-637.

7. Huang C, Wang Y, Li X. et al. Clinical features of patients infected with 2019 novel coronavirus in Wuhan, China. Lancet. 2020; 395:497-506

8. Prockop DJ. The exciting prospects of new therapies with mesenchymal stromal cells. Cytotherapy. 2017; 19:1-8.

9. Connick P, Kolappan M, Crawley C, Webber DJ, Patani R, Michell AW, Du MQ, Luan S, Altmann DR, Thompson AJ, Compston A, Scott MA, Miller DH, Chandran S. Autologous mesenchymal stem cells for the treatment of secondary progressive multiple sclerosis: an open-label phase 2a proof-of-concept study. Lancet Neurol. 2012; 11:150-156.

10. Wilson JG, Liu KD, Zhuo NJ. et al. Mesenchymal stem (stromal) cells for treatment of ARDS: a phase 1 clinical trial. Lancet Respir Med. 2015; 3:24-32.

11. Kamen DL, Nietert PJ, Wang H, Duke T, Cloud C, Robinson A, Gilkeson GS. CT-04 safety and efficacy of allogeneic umbilical cord-derived mesenchymal stem cells (MSCs) in patients with systemic lupus erythematosus: results of an open-label phase I study. Lupus Sci Med. 2018; 5:46-47.

12. Hashmi S, Ahmed M, Murad MH, Litzow MR, Adams RH, Ball LM, Prasad VK, Partow Kebriaei P, Ringden O. Survival after mesenchymal stromal cell therapy in steroid-refractory acute graft-versus-host disease: systematic review and meta-analysis. Lancet Haematol. 2016; 3:45-52

13. Prockop DJ, Oh JY. Mesenchymal stem/stromal cells (MSCs): role as guardians of inflammation. Mol Ther. 2012; 20:14-20.

14. Aggarwal S, Pittenger MF. Human mesenchymal stem cells modulate allogeneic immune cell responses. Blood. 2005; 105:1815-1822.

15. Chan MC, Kuok DI, Leung CY, Hui KP, Valkenburg SA, Lau EH, Nicholls JM, Fang X, Guan Y, Lee JW, Chan RW, Webster RG, Matthay MA, Peiris JS. Human mesenchymal stromal cells reduce influenza A H5N1associated acute lung injury in vitro and in vivo. Proc Natl Acad Sci U S A. 2016; 113:3621-3626.

16. Chen J, Hu C, Chen L, Tang L, Zhu Y, Xu X, Chen L, Gao H, Lu X, Yu L, Dai X, Xiang C, Li L. Clinical study of mesenchymal stem cell treating acute respiratory distress syndrome induced by epidemic Influenza A (H7N9) infection, a hint for COVID-19 treatment. Engineering (Beijing). 2020. 
17. Golchin A, Farahany TZ, Khojasteh A, Soleimanifar F, Ardeshirylajimi A. The clinical trials of Mesenchymal stem cell therapy in skin diseases: An update and concise review. Curr Stem Cell Res Ther. 2019; 14:22-33.

18. Leng Z, Zhu R, Hou W, et al. Transplantation of ACE2mesenchymal stem cells improves the outcome of patients with COVID-19 pneumonia. Aging Dis. 2020; 11:216228.

19. Liang B, Chen J, Li T, Wu H, Yang W, Li Y, Li J, Yu C, Nie F, Ma Z, Yang M, Nie P, Gao Y, Qian C, Hu M. Clinical remission of a critically ill COVID-19 patient treated by human umbilical cord mesenchymal stem cells. ChinaXiv. 2020

20. The Niche. https://ipscell.com/2020/03/athersys- mesoblast-stem-cell-drugs-for-novelcoronavirus-covid-19/ (assessed May 10, 2020).

Received May 15, 2020; Revised May 25, 2020; Accepted June 13, 2020.

*Address correspondence to:

Sangeeta Choudhury, Department of Research, Room No: 1058, Sir Ganga Ram Hospital, Rajinder Place, New Delhi 110060, India.

E-mail: dr.sangeeta.sgrh@gmail.com

Released online in J-STAGE as advance publication June 16, 2020. 